\title{
Erratum: Logarithmic Chain-Exchange Kinetics of Diblock Copolymer Micelles [Phys. Rev. Lett. 96, 068302 (2006)]
}

\author{
Reidar Lund, Lutz Willner, Jörg Stellbrink, Peter Lindner, and Dieter Richter \\ (Received 23 November 2009; published 29 January 2010)
}

DOI: 10.1103/PhysRevLett.104.049902

PACS numbers: 82.70.Uv, 61.05.fm, 61.25.he, 82.35.Lr, 99.10.Cd

In light of new results presented recently by Bates and co-workers [1], we have reanalyzed our data from our Letter published in 2006 [2]. There, we found that the exchange kinetics of a series of diblock copolymer micelles follows a logarithmic time dependence. In order to explain this behavior, we developed a model assuming that the kinetics is dominated by unimer exchange and applying the scaling predictions for the expulsion rate constant by Halperin and Alexander [3]. As this result gives a single exponential decay, we modified the expression by a Poisson distribution function that takes into account the polydispersity of the core-forming polymer. Since the agreement with the experimental data was only poor, we concluded that the polydispersity alone was not sufficient to describe the broad relaxation pattern. However, for the calculation, the prefactor $\alpha$ of the activation barrier term within the scaling approach was simply set to 1 . At that time we did not consider the dramatic effect on the results of small changes of $\alpha$ due to the double exponential dependence. Here we show that the logarithmic time dependence is obtained with a parameter $\alpha$ larger than 1 . The time dependence resulting from this change corroborates the critical effect of polydispersity on the exchange kinetics of polymeric micelles.

Figure 1 shows kinetic data of poly(ethylene-alt-propylene)-block-poly(ethylene oxide) (PEP1-PEO20) micelles in water/DMF mixture. The solid lines represent fits obtained by the original expression for the activation energy but now with a fitted prefactor $\alpha . E_{a}=\alpha \cdot \gamma \cdot N^{2 / 3} \cdot l^{2}$, where $\gamma$ denotes the interfacial tension, $N$ the number of repeat units, and $l$ an effective segment length. In addition, to account for the temperature dependence, we have included an attempt time $\tau$ that scales with the friction coefficient $\xi(T)$ of PEP for a homopolymer melt [4]: $\tau=\tau_{0} \frac{\zeta(T)}{\zeta(47)}\left(\frac{N}{\left\langle N_{n}\right\rangle}\right)^{2 / 25}$, with $\left\langle N_{n}\right\rangle$ the mean number of repeat units.

An almost perfect reproduction of the logarithmic decay of the experimental data is obtained with $\alpha=3.3$ and $\tau_{0}=$ $2.4 \times 10^{-7} \mathrm{~s}$. While the latter value is a typical elemental time expected for polymer dynamics, the value of the prefactor $\alpha$ still needs to be understood. If the core-forming polymer block would form a perfect sphere in the expulsion process, we would expect a value of $\alpha=(4 \pi)^{1 / 3} \cdot 3^{2 / 3} \approx 4$.8. A smaller value may be an indication of a different polymer conformation or interfacial effects due to, e.g., modifications of surface energy by the other block. However, this remains to be further explored in future studies. Thus, from these results, we conclude that-deviating from our initial interpretation-polydispersity of the core-forming polymers, although very low for polymers synthesized by living polymerization, cannot be neglected for the description of the unimer exchange kinetics of diblock copolymer micelles.

This agrees with the main conclusion given by Bates and co-workers [1]. They found a corresponding logarithmic decay in a system very similar to one of the three systems already presented in our letter. Their model considers in addition to

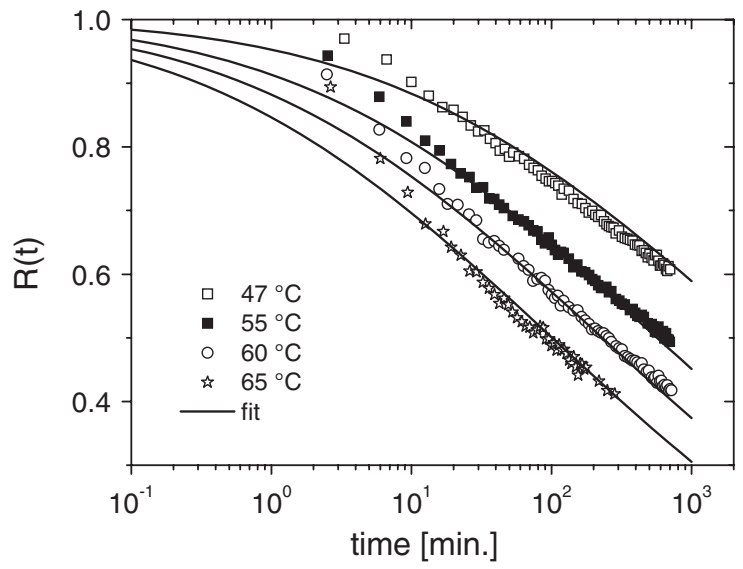

FIG. 1. Chain-exchange kinetics of PEP1-PEO20 micelles in DMF/water solution. The lines represent simultaneous fits of the modified original model. 
adapted prefactors, a slightly different activation energy term where the polymer chains were assumed to be completely stretched in the expulsion process. This leads to an expression of $E_{a}$ which is linearly dependent on $N$.

In summary, we state that our earlier erroneous dismissal of polydispersity as cause for the logarithmic kinetic time dependence came due to neglect of the extreme sensitivity on the proper value of the prefactor $\alpha$. However, to explain the mechanism and the polymer conformations involved in the activated steps in the exchange process, which gives the exact value of $\alpha$, more investigation needs to be done.

[1] S.-H. Choi, T. P. Lodge, and F. S. Bates, this issue, Phys. Rev. Lett. 104, 047802 (2010).

[2] R. Lund, L. Willner, J. Stellbrink, P. Lindner, and D. Richter, Phys. Rev. Lett. 96, 068302 (2006).

[3] A. Halperin and S. Alexander, Macromolecules 22, 2403 (1989).

[4] D. Richter, M. Monkenbusch, A. Arbe, and J. Colmenero, Neutron Spin Echo in Polymer Systems, Advances in Polymer Science (Springer, Berlin, Germany, 2005), Vol. 173. 